\title{
Electrically tunable Bragg gratings in single-mode polymer optical fiber
}

\author{
K. Kalli \\ Nanophotonics Research Laboratory, Higher Technical Institute, Cyprus \\ H. L. Dobb, D. J. Webb, and K. Carroll \\ Photonics Research Group, Aston University, UK \\ M. Komodromos and C. Themistos \\ Frederick Institute of Technology, Cyprus \\ G. D. Peng \\ University of New South Wales, Australia \\ Q. Fang and I. W. Boyd \\ University College London, UK
}

Received September 27, 2006; revised October 25, 2006; accepted November 6, 2006; posted November 8, 2006 (Doc. ID 75503); published January 12, 2007

\begin{abstract}
We present what is to our knowledge the first demonstration of a tunable fiber Bragg grating device in polymer optical fiber that utilizes a thin-film resistive heater deposited on the surface of the fiber. The polymer fiber was coated via photochemical deposition of a $\mathrm{Pd} / \mathrm{Cu}$ metallic layer with a procedure induced by vacuum-ultraviolet radiation at room temperature. The resulting device, when wavelength tuned via joule heating, underwent a wavelength shift of $2 \mathrm{~nm}$ for a moderate input power of $160 \mathrm{~mW}$, a wavelength to input power coefficient of $-13.4 \mathrm{pm} / \mathrm{mW}$, and a time constant of $1.7 \mathrm{~s}^{-1}$. () 2007 Optical Society of America OCIS codes: $060.2340,060.1810$.
\end{abstract}

There are many important applications for tunable fiber devices in optical sensing and lightwave communication systems. The use of fiber Bragg gratings (FGBs) as tunable filtering candidates has been demonstrated in glass fibers with wavelength and bandwidth tunability shown by use of strain $^{1}$ and temperature. ${ }^{2,3}$ FBG filters coated with thin-film heaters on the surface have been promoted as wavelength-tunable devices because of their compact nature, fast response, and high efficiency. It has been noted recently that polymer optical FBGs have a high temperature sensitivity, ${ }^{4}$ and hence they may be suitable candidates for producing a widely tunable filter by heating the fiber. Thermally tuned gratings in polymer waveguides have resulted in large wavelength tuning, but in polymer material that cannot be extruded into fiber. ${ }^{5}$ In this Letter we demonstrate what is believed to be the first wavelength-tunable polymer optical FBG filtering device that relies on thin-film resistive load heating to produce wavelength changes.

FBGs were inscribed into step-index single- and few-mode polymer optical fiber [polymethylmethacrylate (PMMA)] by use of a $30 \mathrm{~mW}$ Kimmon IK series $\mathrm{HeCd}$ laser emitting at $325 \mathrm{~nm}$ by means of the standard phase mask technique. The phase mask had a pitch of $1060.85 \mathrm{~nm}$ and was designed to produce a FBG at $1568 \mathrm{~nm}$ in the polymer optical fiber, in contrast with the $1536 \mathrm{~nm}$ design wavelength for silica fiber. Further details of the inscription procedure may be found in Ref. 6 .

0146-9592/07/030214-3/\$15.00
The surface region around the grating was coated with a $\mathrm{Pd} / \mathrm{Cu}$ metallic layer; the former aided adhesion to the PMMA, and the latter acted as the thinfilm heater element. Metal deposition was initiated by use of a vacuum-ultraviolet (VUV) light source operating at $172 \mathrm{~nm}$ and at low (room) temperature using the arrangement in Fig. 1. At this wavelength the penetration depth of the light source is of the order of $1 \mu \mathrm{m}$, and therefore there is no damage to the grating device, but the surface of the sample undergoes chemical roughening, as the light initiates bond

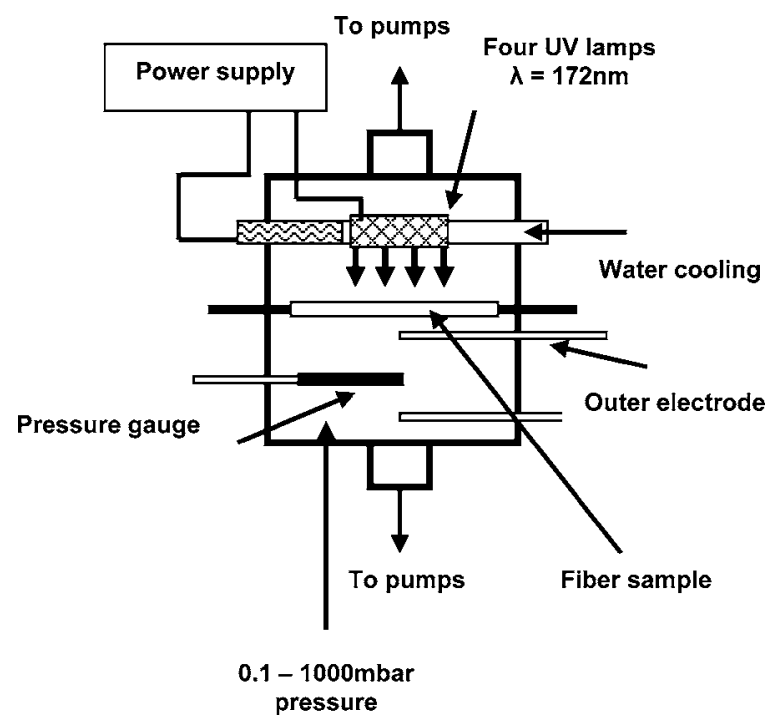

Fig. 1. Schematic diagram of the coating procedure. 


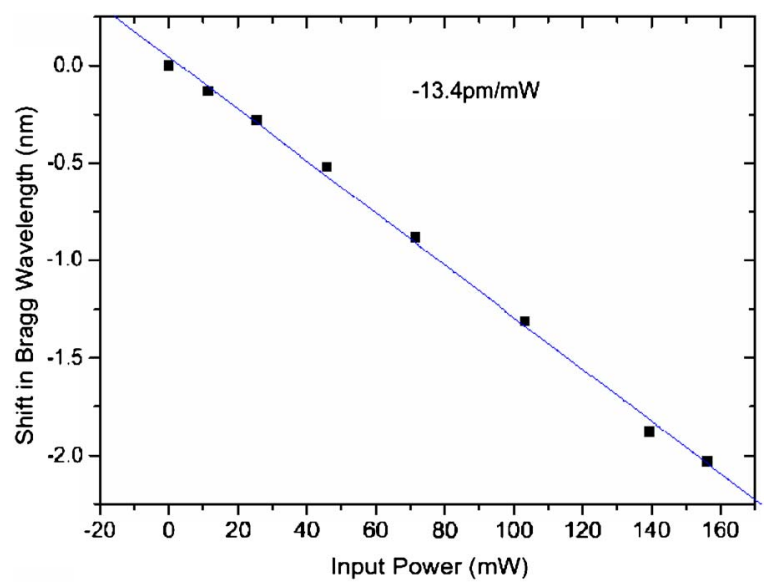

Fig. 2. Wavelength shift induced by joule heating with a wavelength to input power coefficient of $-13.4 \mathrm{pm} / \mathrm{mW}$.

scission. The VUV exposure time ranged from 1 to $10 \mathrm{~min}$. The dangling polymer bonds bond both physically and chemically to metallic particles. This is of great advantage as there are very few approaches for coating polymers with metal at room temperature and much higher temperatures would have an adverse impact on the performance and spectral characteristics of the grating. Furthermore, the coating took place in a low vacuum of $10^{-2}$ mbars, which again is not enough to affect the basic fiber and grating properties. This approach of decomposing metal organic films with incoherent VUV excimer lamps at low temperature is ideal for the manufacture of thin-film microcircuits and devices, proving to be an efficient technique for the preparation of uniform thin films. The film thickness is controlled by variation of the reaction parameters, the concentration of precursor and the UV-irradiation time.

The response of thin-film heated Bragg gratings in glass optical fibers has been characterized, but we anticipate differences between the thermal properties of the glass and polymer-based devices, principally the thermal diffusion and radiation dissipation, given the different thermal conductivities and emissivities of glass and polymer materials. We consider a differential equation that describes the temperature distribution and thermal flow in the fiber, assuming that the coating is sufficiently uniform and greater in length than the grating to give a uniform temperature distribution across the grating length, so only the radial distribution and thermal flow along the grating length are important. The device is modeled as a polymer cylinder with a thin-film thermal source on the surface enclosed by air. In this way the heat diffuses into the fiber core and the surrounding air, in addition to radiation. From energy conservation within the fiber, ${ }^{7}$

$$
\frac{\partial T(x, t)}{\partial t}=\kappa \frac{\partial^{2} T(x, t)}{\partial x^{2}}+\frac{P_{\text {in }}(x, t)-P_{\text {out }}(x, t)}{\rho c_{p} \pi R_{\text {fiber }}^{2}},
$$

where $x$ is the distance along the fiber; $T(x, t)$ is the fiber temperature; $\kappa, \rho$, and $c_{p}$ are the thermal diffusivity, density, and constant pressure heat capacity of the polymer (PMMA), respectively; and $R_{\text {fiber }}$ is the fi- ber radius. $P_{\text {in }}(x, t)$ is the heating generated by the thin-film heater, and $P_{\text {out }}(x, t)$ is the rate of heat loss from the fiber to the air. In general $P_{\text {in }}(x, t)$ $=I^{2}(t) R(x)$, where $R(x)$ is the local resistance per unit length of the coating and $I(t)$ is the electrical current. Furthermore, if we assume that the rate of heat loss is proportional to the change in temperature, then $P_{\text {out }}(x, t)=a\left|T(x, t)-T_{\text {air }}\right|$, where $a$ is a constant that may be temperature dependent and represents a lumped heat transfer coefficient due to heat transfer from the surface of the device. The rate of heat loss is linearly proportional to the change in temperature,

$$
P_{\text {out }}(x, t)=2 \pi R_{\text {fiber }} h T(x, t),
$$

where $h$ is the surface conductance of the fiber, which characterizes the heat flow out of the fiber; additionally,

$$
\frac{P_{\text {out }}(x, t)}{\rho c_{p} \pi R_{\text {fiber }}^{2}}=a T(x, t),
$$

hence $a$ is related to $h$ by

$$
a=\frac{2 h}{\rho c_{p} R_{\text {fiber }}} .
$$

Furthermore, radial heat flow terms have to be considered:

$$
2 \pi R_{\text {fiber }}\left(\kappa \frac{\partial T}{\partial r} r=R_{\text {fiber }}-\kappa_{\text {air }}{\frac{\partial T_{\text {air }}}{\partial r}}_{r=R_{\text {fiber }}}\right),
$$

where $\kappa_{\text {air }}$ and $T_{\text {air }}$ are the thermal diffusivity of air and the air temperature, respectively. This term is important as it accounts for temperature gradients that may exist radially across the grating. Given that the thermal conductivity of PMMA $(0.17 \mathrm{~W} / \mathrm{mK})$ is only 7 times that of air $(0.025 \mathrm{~W} / \mathrm{mK})$, compared with 55 times for silica glass $(1.38 \mathrm{~W} / \mathrm{mK})$, one should consider the validity of ignoring radial gradients and consider only heat flow along the length of the fiber. The heat flow through and along the surface of the fiber is characterized by the Biot number, $\mathrm{Bi}$ $=h R_{\text {fiber }} / \kappa$. If the Biot number is sufficiently small $(<0.1)$, meaning that the thermal conductance over

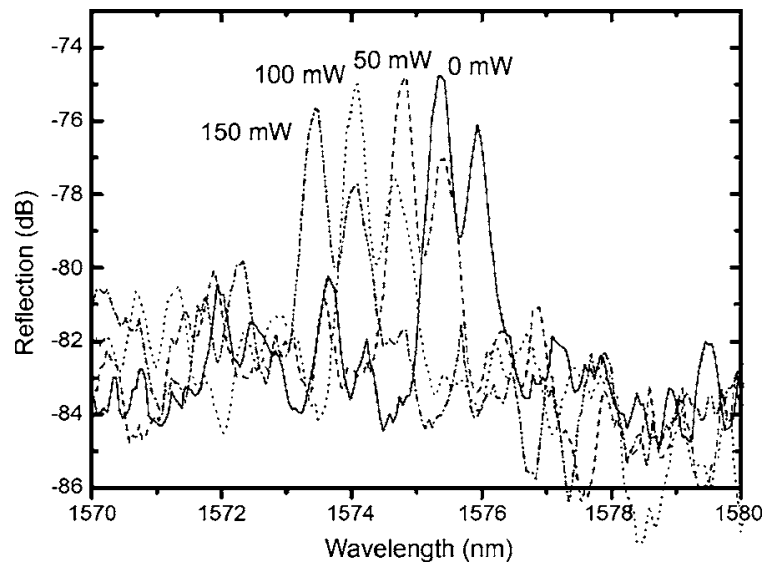

Fig. 3. Wavelength spectrum showing four typical traces for powers of $150,100,50$, and $0 \mathrm{~mW}$. 
the length $R_{\text {fiber }}$ is far greater than the surface conductance, then the radial thermal gradients become negligible. Hence a measurement of $a$ leads to an estimate of $h$, and $\mathrm{Bi}$ and determines whether the fiber can be treated as a 1-D system. $a$ constitutes a system time constant, therefore we must measure the filter's wavelength shift in response to a sudden current input.

To characterize the tunable filter, current was passed through the thin-film metal coating, resulting in joule heating that increased the temperature of the fiber and the grating; any changes in wavelength response due to current flowing through the thin copper film were measured with a broadband light source and optical spectrum analyzer. The total resistance of the film encompassing the grating and the connection via copper wires was measured to be $3.2 \Omega$. Figure 2 shows the wavelength shift induced by joule heating. Note that the wavelength to input power coefficient is negative, as expected, $-13.4 \mathrm{pm} / \mathrm{mW}$. Figure 3 shows the spectrum of the grating for several values of input power. We note that throughout the wavelength tuning the grating spectrum remained unchanged, and this observation is consistent with a spatially uniform heating of the grating. The fiber was nominally single mode at the wavelength of operation, but we observe a spectrum that is dual peaked, a result of a dual-exposure inscription procedure. The grating inscription alters the average core index in addition to the modulated grating component, as the inscription times are relatively long. However, given that the core and cladding are polymerized under different conditions, the core is far more responsive to grating inscription than the cladding region. We have certainly demonstrated good single-mode gratings in both step-index and microstructured polymer optical fibers. ${ }^{6,8}$

When the input power is uniform the temperature variation as a function of time is

$$
T(t)=\frac{P_{\text {in }}^{\prime}}{a}[1-\exp (-a t)]
$$

where $P_{\text {in }}^{\prime}$ is related to the input power but depends on the thermal capacity of the PMMA and the metal film. As noted earlier, a measurement of $a$ yields information regarding the rate of heat flow out of the fiber. Figure 4 shows the rise time of a thermally tuned FBG, with data collected for three different heating powers applied suddenly at $t=0 \mathrm{~s}: 45.8,71.5$, and $103.2 \mathrm{~mW}$. The curves correspond to fits that use a single exponential form, from which we have determined the time constant, $a=1.7 \mathrm{~s}^{-1}$. There is no apparent or systematic dependence on the applied power.

Having determined $a$ we can now evaluate $h$ and $\mathrm{Bi}$. Using the geometric and material properties of the fiber $\left(\rho=1190 \mathrm{~kg} / \mathrm{m}^{3}, \quad c_{p}=1450 \mathrm{~J} / \mathrm{kg} \mathrm{K}, R_{\text {fiber }}\right.$ $=120 \mu \mathrm{m}, \kappa=0.17 \mathrm{~W} / \mathrm{mK}), \quad h$ is found to be $175 \mathrm{~W} / \mathrm{m}^{2} \mathrm{~K}$, and $\mathrm{Bi}$ is $\sim 0.1$; this is compatible with a treatment of the polymer fiber as a 1-D system.

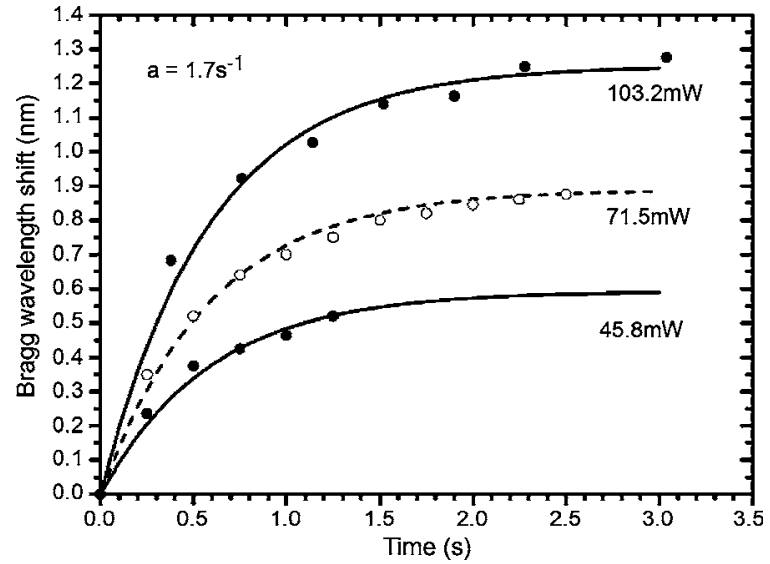

Fig. 4. Rise time of a thermally tuned fiber Bragg grating for three different heating powers applied suddenly at $t$ $=0 \mathrm{~s}: 45.8,71.5$, and $103.2 \mathrm{~mW}$. Time constant $a=1.7 \mathrm{~s}^{-1}$.

We have successfully demonstrated the first wavelength-tunable fiber Bragg grating filter in polymer optical fiber based on thin-film resistive load heating. The device underwent a wavelength shift of $2 \mathrm{~nm}$ for a moderate input power of $160 \mathrm{~mW}$, a wavelength to input power coefficient of $-13.4 \mathrm{pm} / \mathrm{mW}$, and a time constant of $1.7 \mathrm{~s}^{-1}$. A basic study has verified that for this fiber type one can treat the device as a 1-D system. However, as the fiber thickness increases one may have to account for radial gradients across polymer optical fiber based FBG devices. Finally, the coating at room temperature is significant, as there is no adverse impact on the grating spectrum and the procedure is well suited to coating cylindrical polymers with low glass transition temperatures without adversely affecting light guidance.

The authors acknowledge the financial support of the Eureka Project "POLYFILTRO" and the UK Engineering and Physical Sciences Research Council. K. Kalli's e-mail address is kkalli@cytanet.com.cy.

\section{References}

1. A. Iocco, H. G. Limberger, R. P. Salathe, L. A. Everall, K. E. Chisholm, J. A. R. Williams, and I. Bennion, J. Lightwave Technol. 17, 1217 (1999).

2. J. A. Rogers, B. J. Eggleton, J. R. Pedrazzani, and T. A. Strasser, Appl. Phys. Lett. 74, 3131 (1999).

3. B. J. Eggleton, A. Ahuja, P. S. Westbrook, J. A. Rogers, P. Kuo, T. N. Nielsen, and B. Mikkelsen, J. Lightwave Technol. 18, 1418 (2000).

4. H. Dobb, K. Carroll, D. J. Webb, K. Kalli, M. Komodromos, C. Themistos, G. D. Peng, A. Argyros, M. C. Large, M. A. van Eijkelenborg, Q. Fang, and I. W. Boyd, in Proc. SPIE 6189, 1 (2006).

5. L. Eldada, R. Blomquist, M. Maxfield, D. Pant, G. Boudoughian, C. Poga, and R. A. Norwood, IEEE Photon. Technol. Lett. 11, 448 (1999).

6. H. Dobb, D. J. Webb, K. Kalli, A. Argyros, M. C. J. Large, and M. A. van Eijkelenborg, Opt. Lett. 30, 3296 (2005)

7. J. A. Rogers, P. Kuo, A. Ahuja, B. J. Eggleton, and R. J. Jackman, Appl. Opt. 39, 5109 (2000).

8. H. Y. Liu, G. D. Peng, and P. L. Chu, IEEE Photon. Technol. Lett. 14, 935 (2002). 